\section{Band 27, Heft 6, Dezember 2011}

Editorial

415 Kolorektales Karzinom - Standard und neue Wege Piso, P. (Regensburg); Seufferlein, T. (Halle/Saale)

Hauptthema

417 Laparoskopische kolorektale Tumorchirurgie Spatz, H.; Geissler, B.; Paschwitz, R.; Anthuber, M. (Augsburg)

425 Perioperative Therapie des Rektumkarzinoms: Was ist Standard? Wird sich der Standard ändern? Hofheinz, R.-D. (Mannheim); Rödel, C. (Frankfurt/M.)

437 Multimodale Therapiestrategien bei Lebermetastasen kolorektaler Karzinome Stein, A.; Arnold, D. (Hamburg)

445 Metastasiertes kolorektales Karzinom - State of the Art 2011

Reinacher-Schick, A. (Bochum)

455 Zertifizierte Darmkrebszentren - eine Standortbestimmung Benz, S. (Nagold)

Interdisziplinäres Gespräch

461 Kolorektales Karzinom - Standard und neue Wege Schlitt, H.J. (Regensburg); Seufferlein, T. (Halle/Saale); Piso, P. (Regensburg) (Gesprächsleiter)

Klinische Information

465 S3-Leitlinie «Chirurgie der Adipositas»: Kommentierte Kurzfassung

Runkel, N. (Villingen-Schwenningen); Colombo-Benkmann, M. (Münster); Hüttl, T.P. (München); Tigges, H. (Konstanz); Mann, O. (Hamburg)

Fallbericht

473 Rezidivierendes örtlich begrenztes Pseudomyxoma peritonei im weiblichen Becken

Mishin, I.; Ghidirim, G.; Gladun, E.; Mishina, A.; Vozian, M. (Kishinev)

477 News / Ticker

481 Tagungen und Kongresse

486 Inhalt Band 27, 2011

492 Hinweise für Autoren

416 Impressum
Vol. 27, Issue 6, December 2011

Editorial

415 Colorectal Cancer - State of the Art and New Approaches

Piso, P. (Regensburg); Seufferlein, T. (Halle/Saale)

Main Topic

417 Laparoscopic Surgery for Colorectal Cancer Spatz, H.; Geissler, B.; Paschwitz, R.; Anthuber, M. (Augsburg)

425 Perioperative Treatment of Rectal Cancer: What Is Standard? Will the Standard Change? Hofheinz, R.-D. (Mannheim); Rödel, C. (Frankfurt/M.)

437 Multimodal Therapeutic Strategies in Colorectal Cancer Liver Metastases Stein, A.; Arnold, D. (Hamburg)

445 Advanced Colorectal Cancer - State of the Art 2011 Reinacher-Schick, A. (Bochum)

455 Certified Colorectal Cancer Centers - Current Status

Benz, S. (Nagold)

Interdisciplinary Discussion

461 Colorectal Cancer - State of the Art and New Approaches

Schlitt, H.J. (Regensburg); Seufferlein, T. (Halle/Saale); Piso, P. (Regensburg) (Discussion Leader)

Clinical Information

465 Evidence-Based Guidelines for Bariatric Surgery: Commented Short Version

Runkel, N. (Villingen-Schwenningen); Colombo-Benkmann, M (Münster); Hüttl, T.P. (München); Tigges, H. (Konstanz); Mann, O. (Hamburg)

Case Report

473 Recurrent Localized Pseudomyxoma Peritonei in the Female Pelvis

Mishin, I.; Ghidirim, G.; Gladun, E.; Mishina, A.; Vozian, M. (Kishinev)

477 News / Ticker

481 Meetings and Conferences

489 Contents Vol. 27, 2011

492 Guidelines for Authors

416 Imprint

\section{KARGER}

Fax +497614520714

Information@Karger.de

www.karger.com
(C) 2011 S. Karger GmbH, Freiburg 